\title{
Acute Paraplegia with Cognitive Alterations After Bilateral Infarcts in Cerebral Small Vessel Disease
}

\author{
Francisco J Ros Forteza ${ }^{1,2}$ \\ ${ }^{1}$ Neurology Service, Local Health Unit of Guarda, E.P.E., Guarda, Portugal \\ ${ }^{2}$ Department of Medical Sciences, Faculty of Health Sciences, University of Beira Interior, Covilhã, Portugal
}

How to cite this article: Ros Forteza FJ. Acute paraplegia with cognitive alterations after bilateral infarcts in cerebral small vessel disease. EJCRIM 2017;4: doi:10.12890/2017_000697.

Conflicts of Interests: The Authors declare that there are no competing interests.

Acknowledgements: I thank Hugues Chabriat, PhD for his kind help.

This article is licensed under a Commons Attribution Non-Commercial 4.0 License

\section{ABSTRACT}

Cerebral small vessel disease (SVD) affects the small arteries, arterioles, venules and capillaries in the brain and can be identified clinically and/or radiologically. We describe the case of a 71-year-old man with sporadic cerebral SVD who presented with acute paraplegia with urinary incontinence and recent cognitive impairment that developed after the occurrence of ischaemic lesions.

\section{LEARNING POINTS}

- Cerebral small vessel disease (SVD) is extremely common and can mimic severe injury of lower motor neurons.

- Newer imaging techniques have significantly helped in the diagnosis of SVD.

- A combination of bilateral motor and/or sensory deficits located only in the lower limbs should raise the possibility of the bilateral deep lesions that can be observed in cerebral SVD.

\section{KEYWORDS}

Acute paraplegia; mild cognitive impairment; bilateral cerebral small vessel disease

\section{INTRODUCTION}

Cerebral small vessel disease (SVD) affects the small arteries, arterioles, venules and capillaries of the brain ${ }^{[1]}$. SVD is responsible for cerebral small deep infarcts, haemorrhages, microbleeds ${ }^{[2]}$ and white matter lesions.

We here describe a case of acute paraplegia with urinary incontinence and recent cognitive impairment that occurred after the development of ischaemic lesions in a patient with sporadic cerebral SVD.

\section{CASE REPORT}

Three days before admission, a 71-year-old man awoke with complete paraplegia and urinary incontinence. The patient was known to be hypertensive and hyperlipidaemic, and to smoke 10 cigarettes per day. He was being treated with clopidogrel 75 mg and rosuvastatin 5 mg, both once a day.

The patient first underwent a CT scan which revealed lumbar osteoarthritis at L2-4, and disc herniations from L1 to L5 and S1, with root compression at L4. He was then transferred to our emergency room. At entry, his blood pressure was $146 / 74 \mathrm{mmHg}$, and his pulse was 
regular at 66 beats/min. Neurological examination showed complete flaccid paralysis of both legs with abolition of reflexes, pain sensory loss in the right leg, and preservation of proprioception and vibratory sensation in the lower extremities of both legs. The plantar reflex on the left was indifferent and on the right in extension. Neurological examination of the upper extremities was normal. The patient also had obvious cognitive changes with temporo-spatial disorientation and recall difficulties (MMSE scale 24/30: orientation 8, registration 3, attention and calculation 5 , recall 0 and language 8 ).

Two months previously, the patient had experienced an acute ischaemic stroke in the right dorsal callosal artery territory (Fig 1a, arrow) resulting in reduced consciousness, dysarthria, hypoesthesia, imbalance and gait disturbances. The patient had no cognitive complaints and could independently perform activities of daily living. Multiple white matter hyperintensities on FLAIR and T2-weighted images (Fig. 1b,c) were detected bilaterally in the thalamus, basal ganglia, corona radiata and pons (Fig. 1b, arrow).

The work-up included a normal EKG and Holter EKG, and an ultrasound examination showing 30\% stenosis of the right internal carotid artery with a hypoplastic right vertebral artery. Transthoracic echocardiography revealed diastolic dysfunction with left ventricular hypertrophy. Transoesophageal echocardiography was normal. Blood tests (complete cell count, prothrombin time, partial thromboplastin time, electrolytes, NT-proBNP, folate, vitamin B12, thyroid and lipid profiles, uric acid, autoimmune tests, tumour markers and VDRL, hepatitis, and HIV-1 and HIV-2 antibodies) were normal except for the erythrocyte sedimentation rate which was $48 \mathrm{~mm} / \mathrm{h}$.

MRI (Fig. 1d,e) showed two ischaemic lesions. The first was located in the right striatum and caudate and included the anterior limb of the ipsilateral internal capsule (Fig. 1d, arrow) with a discrete haemorrhagic component (Fig. 1e, arrow). The other lesion was located in the left and medial part of the thalamus (Fig. 1e, arrow). Both lesions captured contrast without diffusion restriction. Also, the chronic ischaemic lesion in the right dorsal callosal artery was detected with small thalamic lacunes (Fig. 1f). MRA including a 3D TOF study was normal. Multiple white matter hyperintensities on FLAIR and T2-weighted images highly suggestive of SVD were detected in the centrum semiovale, periventricular areas and brainstem (Fig. 1g). Complete MRI of the spinal cord revealed signs of degeneration in the L4-5 spinal segments with a reduction in interbody space height and Modic type II changes. EMG revealed a bilateral lumbosacral root lesion of moderate severity. CSF was normal. The patient was treated with antiplatelet agents.
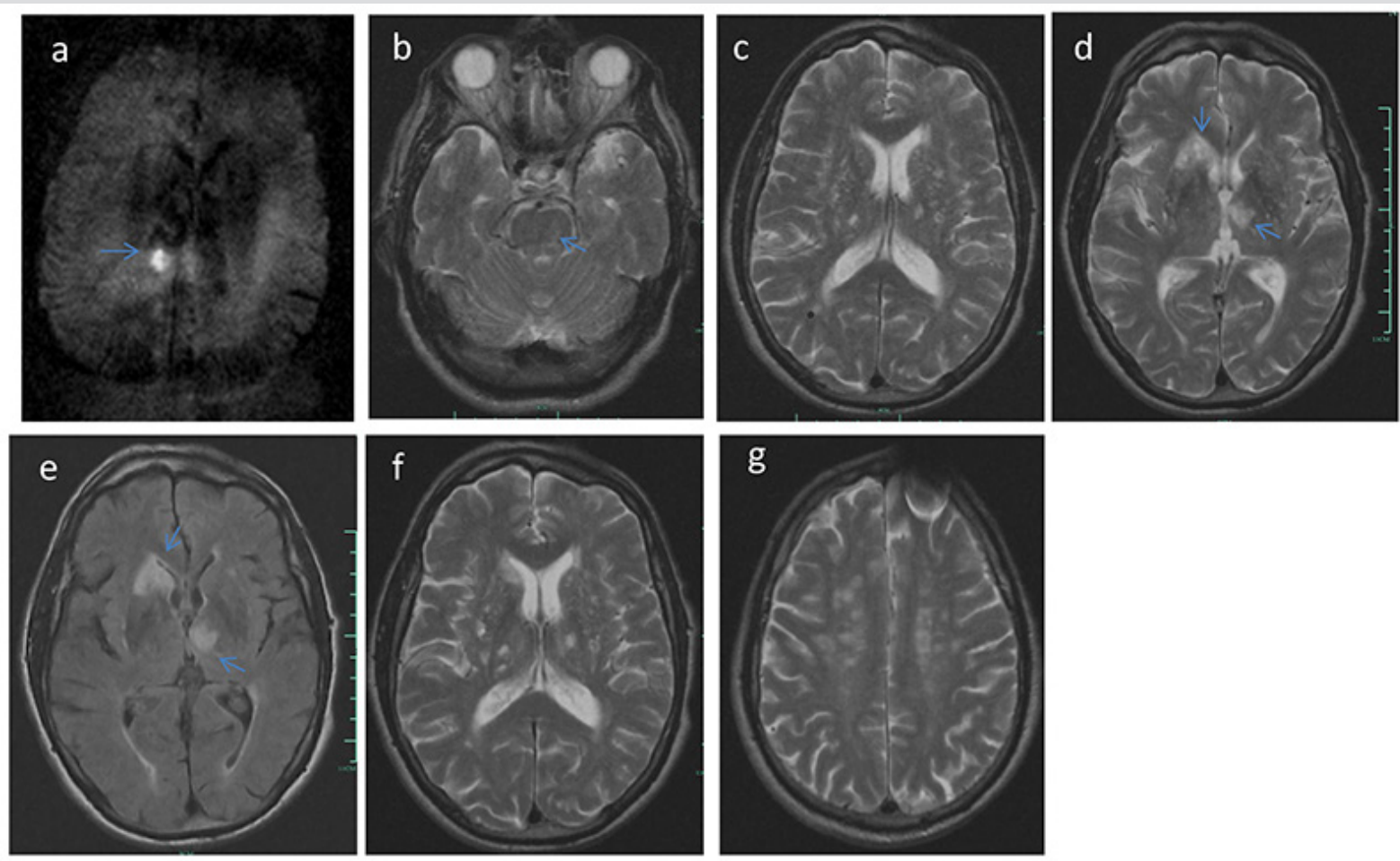

Figure 1. (a) Axial diffusion-weighted MR image showing a right paramedian high lesion in the splenius of the corpus callosum corresponding to an acute ischaemic stroke in the right dorsal callosal artery. (b) Axial T1-weighted MR image showing a high lesion in the pons. (c) Axial T2-weighted MR image showing bilateral white matter hyperintensities in the thalamus, basal ganglia, corona radiata and pons. (d) Axial T2-weighted MR image showing right striatum and caudate high lesions and a left thalamic high lesion. (e) Axial T1-weighted MR image showing a right lesion with a discrete haemorrhagic component and a left thalamic high lesion with mass effect and bulging of the third ventricle. (f) Axial T2-weighted MR image showing a chronic ischaemic lesion in the right dorsal callosal artery with small thalamic lacunes. (g) Axial T2-weighted MR image showing multiple white matter hyperintensities in the centrum semiovale, periventricular areas and brainstem 
After 6 months of rehabilitation with daily mobilization, tactile stimulation techniques and intensive training to improve walking, a substantial improvement was seen: the patient was able to walk and did not have any significant cognitive impairment.

\section{DISCUSSION}

Acute paraplegia associated with cognitive impairment is uncommon in stroke patients. During the acute phase of ischaemic stroke involving the upper motor neurons (UMN), the deficit can mimic that observed after a severe injury to the lower motor neurons (LMN) responsible for flaccid paralysis, reduced tone in the extremities and decreased reflexes. In the present case, the acute paraplegia was initially associated with urinary incontinence and highly suggestive of spinal cord injury although no obvious sensory segmental level was detected. However, no spinal cord injury was seen and the bilateral lumbosacral root compression did not explain the severe deficit that was accompanied by UMN signs. Motor weakness in the left leg was presumably secondary to occlusion of the striate branches of the right anterior cerebral artery leading to an acute insult in the upper and anterior limb of the internal capsule, while pain sensory loss and severe motor neglect in the right leg were presumably related to the thalamic lesion involving both the medial, ventral lateral (VL) and ventral posterolateral (VPL) thalamic nuclei. In addition, a reduction in perfusion of the left thalamus may cause severe and permanent motor neglect ${ }^{[3]}$. Both lesions might affect cognitive performance, as both the striatum and caudate have been previously linked to learning ability and attention ${ }^{[4]}$. Medial lesions in the left thalamus can also significantly alter verbal memory, state of arousal and various cognitive functions ${ }^{[5]}$. Basal ganglia lesions have been also shown to cause urinary incontinence or urinary urgency by promoting detrusor overactivity leading to lower urinary tract dysfunction ${ }^{[6]}$.

Infarcts were responsible for the paraplegia in this patient with moderate deficits related to radiculopathy and recent cognitive alterations. The aetiopathogenic classification of cerebral SVD indicates our patient probably had type 1 arteriolosclerosis (or age-related and vascular risk factor-related SVD) ${ }^{[1]}$.

Finally, this case report suggests that a combination of bilateral motor and/or sensory deficits, although located only in the lower limbs, should raise the possibility of bilateral deep lesions that can be observed in cerebral SVD.

\section{REFERENCES}

1. Pantoni L. Cerebral small vessel disease: from pathogenesis and clinical characteristics to therapeutic challenges. Lancet Neurol 2010;9:689-701.

2. Moran C, Phan TG, Srikanth VK. Cerebral small vessel disease: a review of clinical, radiological, and histopathological phenotypes. Int J Stroke 2012;7:36-46.

3. Manabe Y, Kashihara K, Ota T, Shohmori T, Abe K. Motor neglect following left thalamic hemorrhage: a case report. J Neurol Sci 1999;171:69-71.

4. Liljeholm M, O’Doherty JP. Contributions of the striatum to learning, motivation, and performance: an associative account. Trends Cogn Sci 2012;16:467-475.

5. Edelstyn NM, Ellis SJ, Jenkinson P, Sawyer A. Contribution of the left dorsomedial thalamus to recognition memory: a neuropsychological case study. Neurocase 2002;8:442452.

6. Sakakibara R, Panicker J, Fowler CJ, Tateno F, Kishi M, Tsuyuzaki Y, et al. Vascular incontinence: incontinence in the elderly due to ischemic white matter changes. Neurol Int 2012;4:e13. 